\title{
$\| \mathrm{P} \mid$ \\ UNDERGRADUATE RESEARCH
}

\section{STEM}

\section{Cytotoxicity Evaluation of Surface-Coated Drug Nanocrystals}

Student researcher: Alexandra Seybold, Junior

Many currently utilized chemotherapeutic drugs create considerable toxic side effects when used in the clinic. Research into formulating these drugs as nanocrystals is a developing field with the intent of reducing systemic toxicity while increasing drug efficacy. While pure nanocrystals inherently promote the drug concentration within a tumor, research into whether surface coating of drug nanocrystals enhances treatment efficacy is ongoing. Through experiments utilizing the Sulforhodamine B (SRB) method, we tested various surface-coated paclitaxel nanocrystals to determine any enhancement by the surface coating to the cytotoxicity of the drug. An IC50 (i.e., the drug concentration to inhibit $50 \%$ growth of cancer cells) assay was chosen to determine the cytotoxicity value for each surface-coated formulation on two different cancer cell lines. These in vitro experiments assessed the efficacy of paclitaxel liquid formulation, paclitaxel nanocrystals, and five different, strategically selected polymer-coated paclitaxel nanocrystals that were intended to target overexpressed membrane receptors by cancer cells. It was determined that paclitaxel nanocrystals indeed showed a higher cytotoxicity than the conventional paclitaxel liquid formulation at comparable incubation times. Additionally, polymer-coated nanocrystals had higher cytotoxicity than pure nanocrystals of all concentrations except for the highest tested. The cytotoxicity data is encouraging in that there are more ways to increase cytotoxicity while ideally reducing systemic toxicity for chemotherapy. Both in vitro and in vivo studies are underway to further investigate cellular uptake mechanisms and pharmacokinetics of the surface-treated drug nanocrystals.

Research advisor Tonglei Li writes, “Alexandra's project provided further support for our nanocrystal-based delivery systems for cancer treatment. She was able to determine the cytotoxicity of various surface-treated nanocrystals of a chemotherapeutic agent. The results enabled us to pursuit animal studies helping our research to be translated into clinical applications."

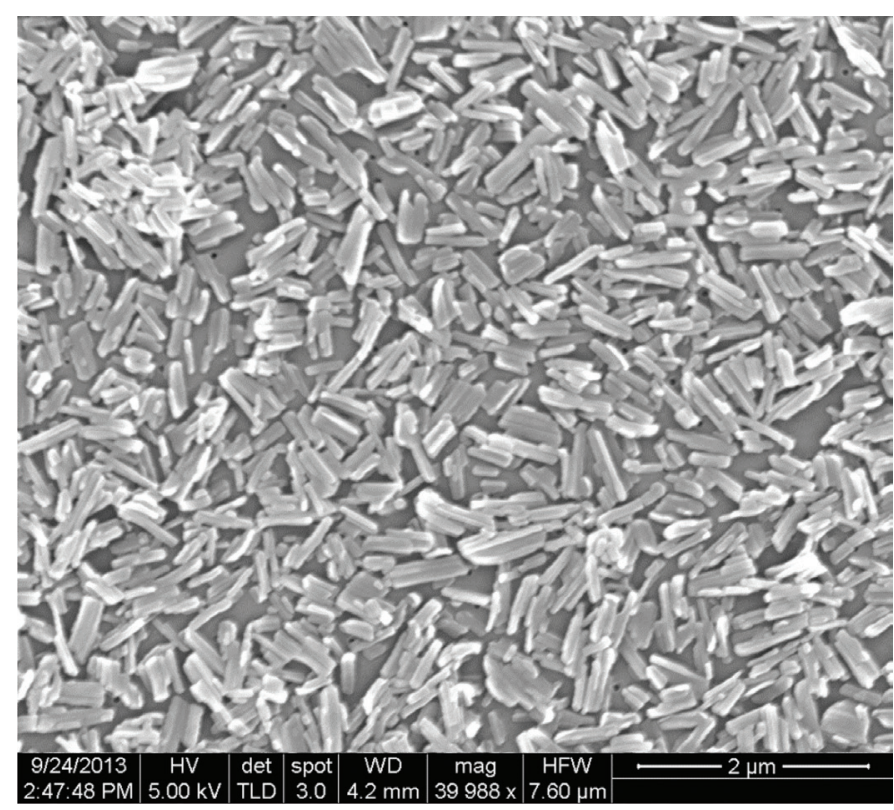

SEM Image of nanocrystals (courtesy of Yan Chen).

Seybold, A. (2015). Cytotoxicity evaluation of surface-coated drug nanocrystals. Journal of Purdue Undergraduate Research, 5, 93. http://dx.doi.org/10.5703/1288284315671 